\title{
Table model and portable optical sensors for the monitoring of time-dependent liquid spreading over rough surfaces
}

\author{
Benjamin Osae Asamoah ${ }^{*}$ (I), Boniphace Kanyathare and Kai-Erik Peiponen
}

\begin{abstract}
Background: Many applications require liquids to efficiently wet required surfaces as it denotes better performance. The dynamics of pure and complex liquid is known to influence the spreading properties; however, this influence is less understood and solicits other measuring techniques to elucidate the grey area. In this work, we demonstrate the use of simple yet novel optical methods in the monitoring of liquid spreading of pure diesel and kerosene and their binary (complex) mixtures.

Methods: The optical devices are a table model and portable optical sensors which use a diffractive optical element for filtering the specular reflection from a laser speckle pattern obtained from the liquid spreading on the rough surface-liquid-air system. The surfaces used in this study were metal surface roughness standards and roughened glass surface. The viability of the devices was demonstrated using two liquids, namely diesel oil and kerosene, that have a wide difference in their contact angles. The performance of the devices was further tested using binary mixtures of the diesel oil and kerosene. Based on the scattering properties of the spreading liquids and the surfaces, the time-dependent signal was measured with the optical devices.

Results: It was observed that the spreading was influenced by the surface roughness. The magnitude of the signal decreased with increased surface roughness indicating less variation in the spreading of the liquid drop with the increased surface roughness. The nature of the detected signal for the kerosene on the surface with roughness values below the wavelength of the device follows the Tanner's law of drop spreading. However, the diesel spreads at a lower rate. Additionally, the complexity of the internal interaction of the diesel-kerosene binary mixtures leads to a complex spreading mechanism on the solid surfaces allowing us to screen the adulterated liquids from the authentic diesel oil with high reliability.

Conclusion: We have introduced two novel optical sensors (table model and portable) for the detection of the changes in liquid drop spreading over rough surfaces. The spreading of the liquid drops over a rough surface causes a local contact angle that experiences hysteresis during the spreading process. The spreading depends on the complexity of the liquid and the magnitude of the surface roughness. The unique configuration of the devices makes the portable sensor suitable for longer duration measurements and for field applications, whilst the table model is best suited for monitoring the first transient moments of liquid spreading and for laboratory applications. Such spreading techniques can also be utilized in the detection of wine and strong alcohol, such as vodka, adulterations with glycol and water, respectively.
\end{abstract}

Keywords: Optical sensing and sensors, Surface dynamics, Roughness, Fuel adulteration

\footnotetext{
*Correspondence: benjaasa@uef.fi

Department of Physics and Mathematics, University of Eastern Finland, PO Box 111, Fl-80101 Joensuu, Finland
} 


\section{Introduction}

Liquid-surface interaction plays a vital role in many applications, for example, in oil lubrication, printing and coating, hydrodynamic properties of aluminium in the marine environment, and in painting. Due to this significance, it has received much attention, especially, in surface engineering. The characterization of the liquid-surface interaction resulting in wetting or spreading of the liquid on the solid surface is determined by the measure of the contact angle. This angle is in turn influenced by the cohesive and adhesive forces at play. On a more fundamental level, material interaction is estimated by Hamaker's constant which is based on the Van der Waals interactions [1], from which the contact angle can also be estimated.

For rough surfaces, the contact angle becomes dynamic and a local quantity during the spreading of a liquid drop and is a function of the surface roughness and other material properties such as the surface tension and viscosity of the liquids. The influence of the surface roughness on the wetting has also received much focus from researchers as demonstrated in several studies [2-10].

In addition to the influence of the surface characteristics on the dynamics of the contact angle, the complexity of the liquid, namely its chemical composition, also plays a critical role on the rate of spreading [11-14]. Spreading of pure liquids on smooth surfaces tends to follow Tanner's law [15] whereas the introduction of surfactants to pure liquids makes the mixture complex, which either enhances or reduces the rate of spreading $[11,14]$.

In this study, we introduce two optical sensors with novel methods for the detection of liquid spreading. The first method is based on rough metal surface standards with different average surface roughness, and the second method is based on a rough glass surface. The use of the metal surface roughness standards provides a common means for the monitoring of liquid drop spreading. The optical detection devices utilized in this work can generally be used for the studying of different liquid spreading, but here we demonstrate the performance of the devices for diesel oil, kerosene, and their binary mixtures which imitate adulterated diesel oils. This method of liquid drop optical monitoring differs from our previous approach [16]. There, we used a robust and portable handheld optical sensor for the screening of adulterated diesel oils at two different time instances (1 and 20 mins). However, in this work, our focus is on the dynamics of the measured signal over different rough surfaces during the initial stage of the drop spreading, and from 1 to 20 mins. These two methods, when combined, give information on the very first minute of the liquid spreading, and up to the $20 \mathrm{~min}$. Moreover, we indirectly obtain information on the hysteresis of the contact angle [2-10].

\section{Materials and method}

We present two optical sensors for studying liquid drop spreading on rough surfaces, namely, the portable sensor (PS) and the table model (TM) (MGM-Devices, Finland) to detect the time-dependent signal (S) from kerosene, diesel oil and their binary mixtures on rough surfaces with different finishing and average surface roughness. Here, the data for ground surface finishing is presented. The TM was used for measuring the drop spreading on metal surfaces for the first minute, whereas the PS was used for measuring the spreading of the liquids on the glass surface at much longer duration, 1-20 min. We briefly consider the liquids, surfaces and the sensors.

We have studied the drop spreading of a high-grade kerosene (Alfa Aesar, Haverhill, MA, USA), authentic diesel oil (diesel grade with crude from Russia), and their binary mixtures. The binary mixtures are also referred to as adulterated. Low-concentration of the kerosene (5-15\%), which is below the typical adulteration levels $(20 \%-30 \%)$, were introduced to the authentic diesel oil and were uniformly mixed to obtain their binary mixtures. The different concentrations of the kerosene in the diesel oil result in the variation in the internal interaction of the admixtures. The interaction of molecules in binary mixture, i.e. chemical activity of liquids, manifests in the optical properties of the binary mixture that is reflected in properties such as the refractive index and extinction coefficient of the binary mixture [17]. We have also shown the failure of the common Lorentz-Lorenz law, typically used for the description of optical properties of binary mixture, for adulterated diesel oils [18]. Furthermore, the chemical activity also reflects in the contact angle and wetting of surface. In some mixtures, such as observed in this study, the interaction is more pronounced in the chemical activity than the optical properties. For these samples, we see the effect of the interaction in the third decimal refractive index (RI) change. The measured RI and the average contact angles (KSV Instruments Ltd., Finland) over five measurements are illustrated in Table 1. The RI of the liquid samples was measured under laboratory conditions using the table model Abbe refractometer (Atago RX5000, Atago Co. Ltd., Tokyo, Japan) at $589 \mathrm{~nm}$ with an accuracy of \pm 0.00004 . The $\mathrm{D}+10 \% \mathrm{~K}$ shows an abnormality in the measured contact angle considering that of the $\mathrm{D}+5 \% \mathrm{~K}$ and $\mathrm{D}+15 \%$. We consider the influence of the contact angle abnormality of $\mathrm{D}+10 \% \mathrm{~K}$ on the signal later in the results and discussion section.

The drop spreading of the liquids was considered for four nickel metal (with RI of $1.98+3.74 \mathrm{i}$ at of $635 \mathrm{~nm}$ [19]) surface standards with different average roughness. 
Table 1 The refractive indices of the liquid samples measured with the table model Abbe refractometer at $589 \mathrm{~nm}$, and the corresponding contact angles measured on a smooth surface

\begin{tabular}{lll}
\hline Sample & $\begin{array}{l}\text { Refractive } \\
\text { index }(n)\end{array}$ & $\begin{array}{l}\text { Contact } \\
\text { angle (deg.) }\end{array}$ \\
\hline Diesel (D) & 1.46373 & 25.9 \\
Kerosene (K) & 1.44230 & 11.2 \\
$D+5 \% K$ & 1.46269 & 22.5 \\
$D+10 \% \mathrm{~K}$ & 1.46163 & 24.7 \\
$D+15 \% \mathrm{~K}$ & 1.46060 & 21.1 \\
\hline
\end{tabular}

The metal standard is a pocket-sized Flexbar composite (FLEXBAR NO. 16008 with ANSI B46.1 standard, Rupert and Co. Ltd.) with ground (GF) finishing. The average roughness values of the studied surfaces are $\mathrm{Ra}=$ $0.05 \mu \mathrm{m}, 0.1 \mu \mathrm{m}, 0.2 \mu \mathrm{m}$, and $0.4 \mu \mathrm{m}$, below and around the wavelength of the laser source. These roughness standards provide a common platform for the monitoring of liquid spreading. As a comparison, similar experiments were carried out on a rough and smooth (on both sides) glass slides (VWR microscope slide ECN 6311550) with RI of 1.4570 at $635 \mathrm{~nm}$ as the spreading surface for the PS. The rough glass has one of its surfaces roughened with a diamond grinding pad and the measured average roughness, $\mathrm{Ra}=0.48 \mu \mathrm{m}$, was determined with the stylus profilometer. Both smooth and rough slides have a $25 \mathrm{~mm}$ diameter and $3 \mathrm{~mm}$ thickness. The ground surfaces allow us to describe the distribution of the roughness height as Gaussian [20]. Hence, the detected reflected light signal from a rough solid-air interface is also a Gaussian function [21, 22], and it depends on the angle of light incidence, the wavelength of the laser light and the average surface roughness. This Gaussian distribution of the reflected signal is accurate for the plane waves. However, the use of a focused laser beam as the light source and the spreading of the liquid drop over the rough surface makes the Gaussian description more complicated. The laser light is scattered both from the rough surface-liquid and the liquid-air interfaces that are in dynamic change. Hence, the speckle pattern in the far-field region is also a dynamic speckle pattern [23]. This dynamic speckle pattern is incident on the diffractive optical element (DOE) which reconstructs the dynamic speckle field. The DOE is a computer-generated hologram fabricated with the electron-beam lithography. It has been used in various applications including the determination of refractive indices of liquids, and a variety of optical inspection of surfaces such as metals, float glass, crystals, ceramics, etc. [24].

A qualitative picture of the specular reflection regarding a stable liquid over the rough glass and metal surfaces can be given using the model of Niskanen et al. [25] as follows:

$$
\mathrm{S} \cong \exp \left\{-\left[\frac{2 \pi R_{a} \cos \alpha}{\lambda}\left(n_{\text {solid }}-n_{\text {liquid }}\right)\right]^{2}\right\}
$$

This model shows how both surface roughness (Ra) and the refractive index difference $\left(n_{\text {solid }}-n_{\text {liquid }}\right)$ of the solid surface and the liquid are coupled together. $n$ is the refractive index, $\lambda$ is the wavelength of the incident light, and $\alpha$ is the angular distribution of the reflected light from the surface. $S$, therefore, describes the specular reflection for both TM and PS, However, for TM, the measurement geometry is at normal incidence.

The schematics of the TM and the PS sensors are illustrated in Fig. 1. The TM is equipped with a sample stage movable in the $\mathrm{x}-\mathrm{y}$ direction at a user-defined resolution in both directions. It is also connected via USB to a PC with software for the recording of the detected signal, and the adjustment of parameters such as power and stage position. In the z-direction, we can also adjust the optics head to perform measurements for different sample thickness.

Both sensors (PS and TM) are the modified versions of the glossmeters which have been described in [26]. Here, we briefly describe the optical features. The TM sensor, as shown in Fig. 1a, consists of a $635 \mathrm{~nm}$ semiconductor laser source, with adjustable power (0$250 \mathrm{~mW}$ ), at normal incidence and the beam is focused to $30 \mu \mathrm{m}$ via a focal lens. We have used $50 \mathrm{~mW}$ power of the laser in this work. The scattered light from the sample is directed with the beam splitter (BS) to the DOE spatial filter which passes the resulting intensity onto a single-cell photodetector. Due to the translation sample stage, the TM can be utilized to scan sample area or to perform fixed point measurements, the latter was applied in this study in a novel way regarding monitoring of a dynamic liquid layer over a rough metal surface. The sensor head and the sample stage were firmly fixed on a vibration-free table.

The PS provides a more compact and portable version for monitoring the liquid spreading with slightly unique features. It uses similar but low-power semiconductor laser $(0.8 \mathrm{~mW})$ light source with 6 degrees angle of incidence and detection, as shown in Fig. 1b. Additionally, the beam is focused to $50 \mu \mathrm{m}$. Despite the wireless infrared connection to a laptop, it only allows for the collection and transmission of 1000 data points in $5 \mathrm{~s}$. Without the laptop, the PS can also be used, but instead, a single average value of the signal is obtainable from the device as it was utilized in [16]. In field application, the PS only needs to be supported mechanically and levelled in the $x-y$ plane. In terms of mode of signal detection, the PS again differs from TM. The PS detects the 


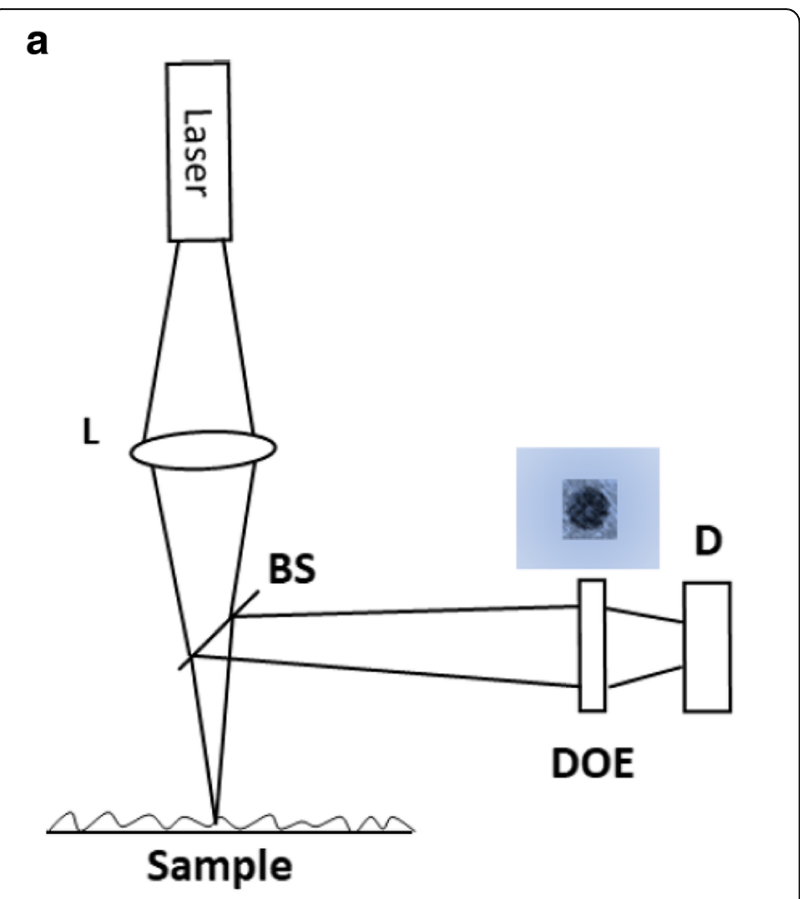

b

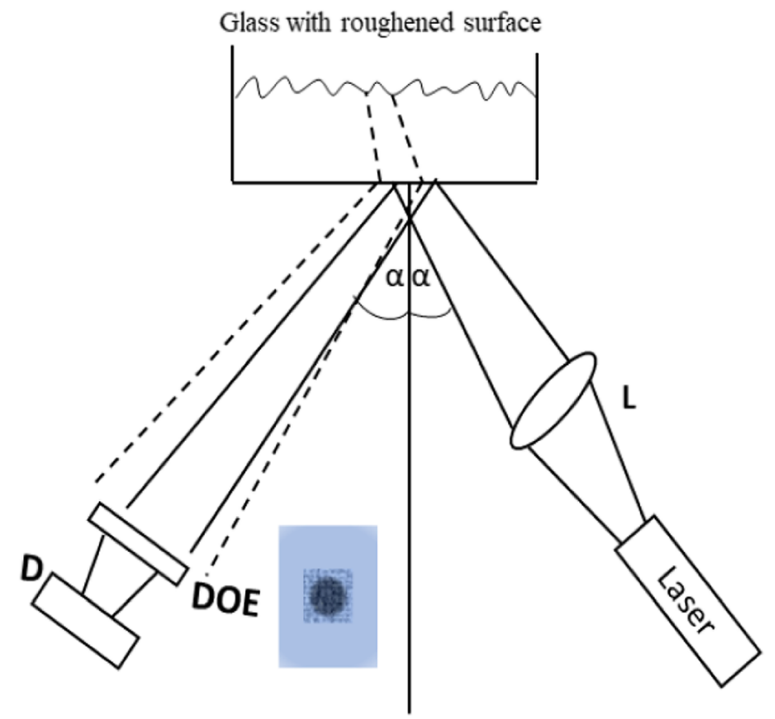

Fig. 1 The schematics of the optical sensor (a) Table model (TM), (b) portable (PS). $L$ is the focusing lens, BS is the beam splitter, $D$ is the single-cell photodiode detector, and $a$, the angle of incidence. The insert is the diffractive optical element (DOE) spatial filter

signal from the spreading liquid through the glass disk whilst the TM detects directly from the upper surface of the rough metal surface.

A drop of $5 \mu \mathrm{L}$ volume of the various liquids was gently introduced by a digital pipette to the metal surfaces at 45 degrees, due to space limitation in the z-direction. Continuous measurement of the spreading at a fixed position was performed for $60 \mathrm{~s}$ after the device calibration. Calibration can be based on using a black glass gloss standard or a planar mirror. After each set of measurements, the surfaces were cleaned several times with ethanol to ensure that new measurements were not affected by the previous samples. For the PS, the measurements were recorded for 20 mins at a 1-min interval for both the rough and smooth surfaces. In both sensors, PS and TM, a small area of the surface-liquid interaction were studied, $50 \mu \mathrm{m}$ and $30 \mu \mathrm{m}$, respectively. Combining both timescales of measurements give a complete view of the drop spreading.

\section{Results and discussions}

The curves depicting the influence of the surface roughness on the spreading of the liquids on both the metal and glass surfaces are presented. The feasibility of the different optical sensors for screening diesel oil and the binary mixtures with low concentration of the adulterant (kerosene) is also demonstrated. The portability and the ease of use make the PS sensor more applicable in field application whilst the TM is rather suited to the laboratory conditions. These optical sensors are useful for the monitoring of dynamic change in the liquid drop spreading which is important in the study of hydrophilic or hydrophobic properties of liquid over rough surfaces. The results of the dynamic signal from the TM are shown in Figs. 2 and 3, and that of the PS in Fig. 4. From the signal obtained using the TM in Figs. 2 and 3, there is abrupt change and fluctuations which are due to the hysterical behavior of the sample depending on the magnitude of the average surface roughness. Such fluctuations are also present in the signal from the PS but less obvious due to its lower resolution.

Figure 2 shows the effect of the metal surface roughness on the spreading of the kerosene and diesel oil. The signal strength is influenced by the magnitude of the average surface roughness. Increasing the surface roughness comparable to the wavelength of the incident light decreases the detected signal strength. The decreasing of the signal also correlates with the spreading of the liquid. This observation is consistent with study [8] where the decreasing of surface roughness increased the spreading of the liquid. The decrease in the signal with the surface roughness could be due to the role of diffuse reflectance. Higher surface roughness leads to increased diffuse reflection consequently decreasing the intensity of the detected signal. The liquid-surface interaction, however, contributes to the growth of the detected signal with time. The top and bottom (in contact with roughness) surface of the liquid spread at different rates with the bottom being impeded by the solid surface roughness. This leads to a planar top surface, increasing the specular reflection (signal) with time as the drop spreads. On the contrary, increasing roughness with the same liquid volume, decreases the planarity of the top surface hence reducing the specular reflection from both the top of the liquid surface and from the metal surface. 


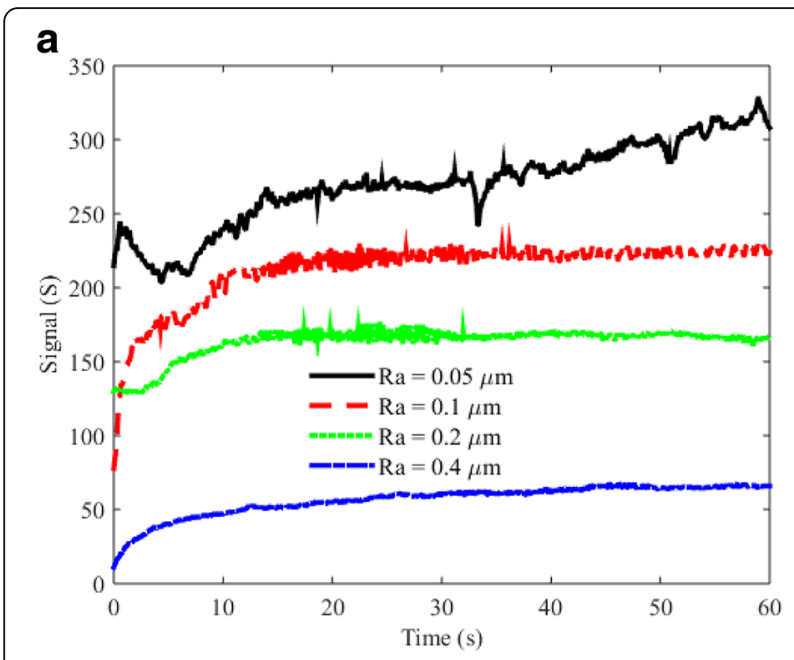

b

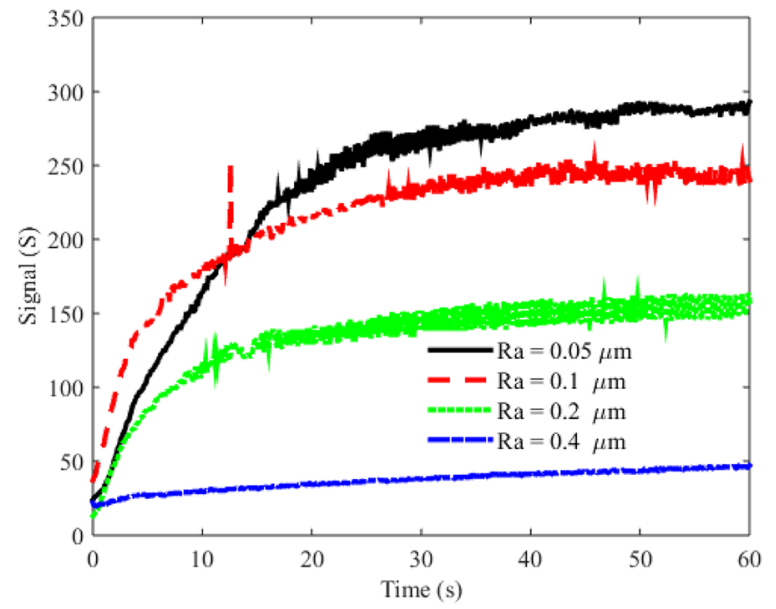

Fig. 2 The dynamic signal (S) of the liquid samples recorded by the TM sensor. a Kerosene (K), (b) for the authentic diesel oil (D). Signal strength increases with time but decreases with the increase in metal surface roughness

Interestingly, averaging and fitting of the signal variation with time by a power function $\left(A x^{b}\right)$ reveals that the growth of the signal agrees with Tanner's law [15] of drop spreading. This was true for kerosene for the average roughness of $\mathrm{Ra}=0.05-0.2 \mu \mathrm{m}$, with $\mathrm{b} \approx 0.1$. Above these roughness values, the spreading can be approximately described by $R(t) \approx \sqrt[4]{t}(2)$. That of the diesel oil can also be described by Eq. (2). $R(t)$, therefore, describes the specular reflection qualitatively defined by $\mathrm{S}$, the signal obtained by both TM and PS, in in Eq. (1). From the dynamic signal, both liquids, kerosene on higher average surface roughness and diesel oil on all the metal surfaces, have a much slower spreading rate with $b \approx 0.2$ compared with kerosene on the surface for $\mathrm{Ra}<0.2 \mu \mathrm{m}$. Thus, from the detected time-dependent signal, we can deduce that, for constant surface roughness, the kerosene spreads faster on the relatively smooth metal

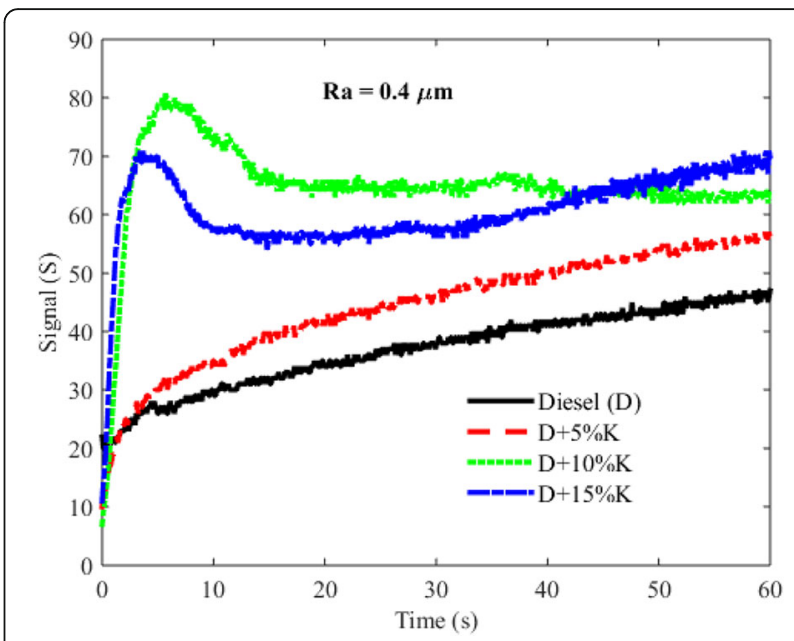

Fig. 3 The dynamic signal (S) of the authentic diesel (D) and the binary mixtures $(D+K)$ recorded by the TM sensor for the average roughness of $\mathrm{Ra}=0.4 \mu \mathrm{m}$

surface than the pure diesel oil. This observation is valid considering the lower contact angle of 11.2 degrees of kerosene. On a smooth surface, therefore, the kerosene is expected to spread much faster than the diesel oil with 25.9 degrees contact angle. The optical signal also exhibits fluctuations with time. These fluctuations can be due to the dynamics of the contact angle between the liquid and the surface. Such hysteresis is apparent in the signal of kerosene for $\mathrm{Ra}=0.05 \mu \mathrm{m}$. Due to the faster spreading rate, kerosene, perhaps, sees the small but abrupt variations in the surface roughness. On the contrary, the diesel with lower spreading shows a smoother signal for the same roughness.

Additionally, at the high surface roughness, the nature of surface finishing could also influence the direction of liquid spreading [8]. Horizontal or vertical ground surface may act as a rail confining the liquid spreading in one direction whilst their combination may otherwise influence in a somehow uniform manner in other directions.

As an example, we have illustrated the dynamics of the signal from authentic and the binary mixtures over the ground metal surface finishing with an average surface roughness value of $\mathrm{Ra}=0.4 \mu \mathrm{m}$, comparable to the laser wavelength, in Fig. 3. The different liquids behave differently on the same surface, giving us the opportunity to screen the adulterated from the authentic diesel oil.

Increasing the concentration of the kerosene in the authentic diesel oil increases the signal. The strength and behavior of the detected signal with time are affected by the wettability of the surface (surface properties) and the liquid. From the dynamic signal, we can deduce that the different liquids namely, the authentic and adulterated diesel oils, therefore, follow a different 


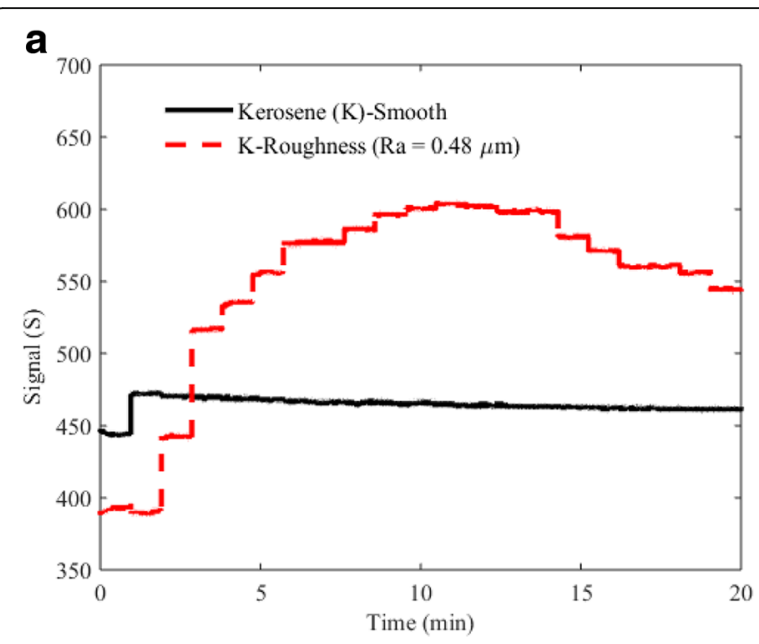

b

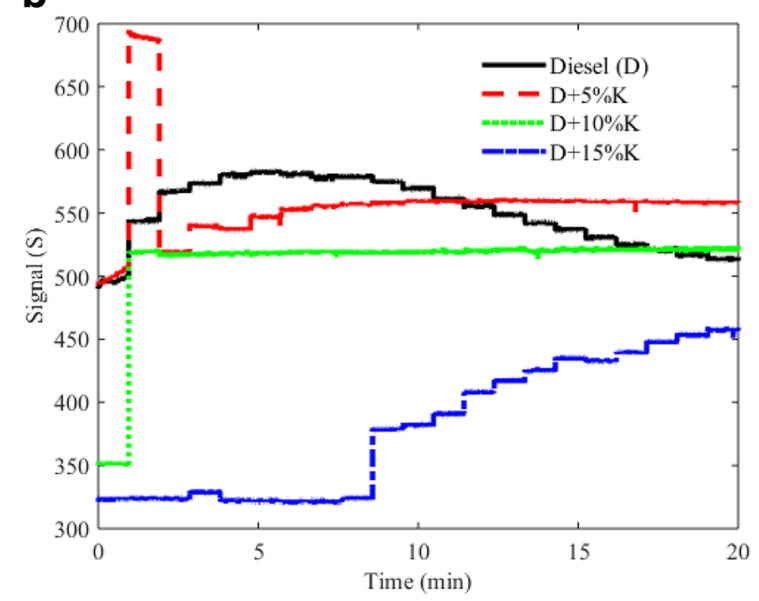

C

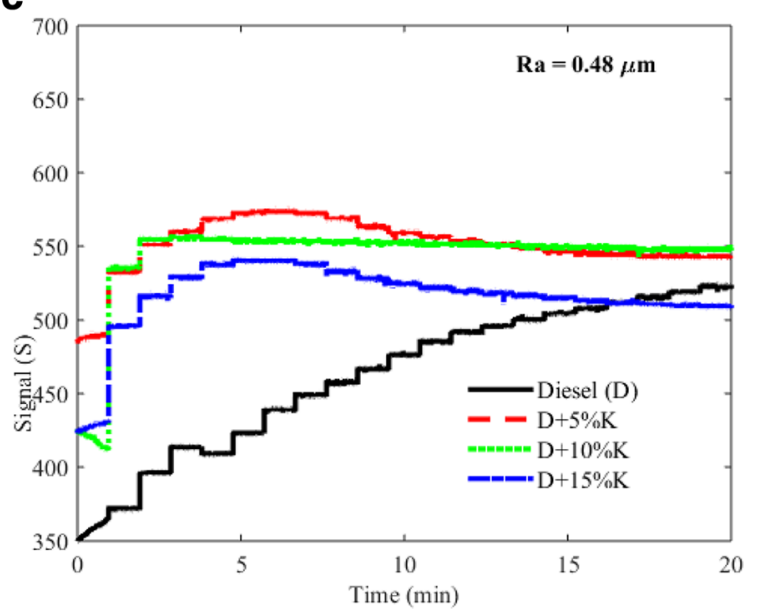

Fig. 4 The dynamic signal (S) from different liquids recorded by the PS. a Kerosene (K), (b) authentic diesel oil (D) and the binary mixtures for the smooth surface. $\mathbf{c}$ authentic diesel oil and binary mixtures $(D+K)$ for the rough glass with $R a=0.48 \mu \mathrm{m}$ spreading mechanism on the rough surface. The introduction of the kerosene influences the chemical as well as the optical properties of the binary mixtures [1] making the liquids complex. For the authentic diesel oil (D) and that with the low concentration of kerosene ( D + $5 \% \mathrm{~K})$, the behavior of the signals with time is very similar to each other, however, with increased signal strength for the $\mathrm{D}+5 \% \mathrm{~K}$. Increasing the kerosene concentration further leads to interesting behavior of the signal. The signal for $\mathrm{D}+15 \% \mathrm{~K}$ is lower than that of $\mathrm{D}+10 \% \mathrm{~K}$. Interestingly, they both show similar nature, sharp initial rise and fall, and remaining somehow constant thereafter. This suggests a faster change in spreading during the initial time duration, compared with the $\mathrm{D}+5 \% \mathrm{~K}$ and the authentic liquids in Fig. 2.

The presence of the kerosene in the diesel oil, therefore, influences the spreading of the liquids on the rough surface due to the interaction of the molecules of the different liquids [16-18, 27]. However, the change in the internal interaction of the adulterated diesel is less evident in the refractive indices of the liquids as illustrated in Table 1. On the contrary, the complexity of the internal interaction of the different molecules of the binary mixtures results in a more obvious abnormal change in the contact angle and a consequent irregularity in the spreading over the external surface. This effect is observed in the corresponding signal from the liquids spreading on the rough surface with the concentration of kerosene greater than $5 \%$. Evidently, with the lower contact angle and lower signal, D $+15 \% \mathrm{~K}$ rather spreads much slower than the $\mathrm{D}+10 \% \mathrm{~K}$ on the highest surface roughness. Such influence of adulterant on liquid spreading has also been observed in other studies [11-14] where surfactants enhance or slow down the spreading rate of the liquid drop on smooth surfaces. In this case, the spreading of the complex liquid changes with the increasing concentration of the adulterant.

The time-dependent signal for the smooth and rough $(\mathrm{Ra}=0.48 \mu \mathrm{m})$ glass slides with the portable sensor (PS) for 1-20 min are shown in Fig. 4. Referable to the two surfaces of the glass slides, the signal also includes contributions from the multiple reflections from the interfacial boundaries unlike in the case of the TM. Additionally, the signal shows steps corresponding to the time-lag $(1-\mathrm{min})$ in the measurement where the contact angle has accumulated hysteresis.

The signal from the kerosene plateaus with time on the smooth surface, whereas the detected signal is evidently influenced by the rough glass surface in Fig. 4a. This suggests a constant spreading of the kerosene on the smooth surface. A similar influence of the surfaces on the spreading of liquid is also observed in the signal from the diesel oil and its binary mixtures for the PS as shown in Fig. 4a and b . 
A more interesting feature is the position of the signal from binary mixtures in comparison to that of the authentic diesel oil on all the surfaces: metal Fig. 3, smooth glass Fig. 4b, and rough glass Fig. 4c. We note that the dynamic signals from the binary mixtures are higher in the TM for the metal surfaces and PS for the rough glass slide than authentic diesel oil. Conversely, the signals for the binary mixtures are lower than that of the authentic diesel oil for the PS of the smooth glass surface. However, as one would expect, the authentic diesel oil has a higher signal for the smooth surface than the rough surface which has its signal growing over time due to the increased specular reflection from the smooth surface.

As seen in Fig. 4b, increasing the concentration of kerosene correspondingly decreases the signal and, consequently, the spreading of the binary mixtures on the smooth surface. Nevertheless, the contact angle suggests otherwise. The contact angle becomes abnormal for the $\mathrm{D}+10 \% \mathrm{~K}$ and, as such, the signal rather correlates with the increasing concentration of the kerosene than with the contact angle. The signal on the smooth surface also shows some hysteresis as have been observed in [28-30]. For the smooth glass surface, the spreading of the authentic diesel oil may suggest a changing shape of a drop to act as a plane or curved mirror. Therefore, the adulterated diesel oil with different concentration of the kerosene adulterant could be forming complex shapes of a mirror on the smooth surfaces.

In Fig. 4c, we see the reverse of the signal behavior of the diesel oil and the binary mixtures on a rough glass slide with average surface roughness, $\mathrm{Ra}=0.48 \mu \mathrm{m}$, comparable to the incident wavelength. Decreasing the concentration of the kerosene leads to the increasing signal from the binary mixtures, but they are, however, higher than the reference authentic diesel oil.

The contact angle of $\mathrm{D}+10 \% \mathrm{~K}$, which characterizes liquid spreading, is abnormal and this abnormality is seen in the signals of PS and TM which show plateaus for different materials (glass and nickel, respectively) with almost the same average surface roughness value of glass and nickel. This implies that the material type and surface roughness have little influence on the spreading of this particular liquid, but instead the contact angle (abnormal) plays a major role. Considering the similarity in the signal behavior of kerosene on smooth surface (Fig. 4a) and that of D $+10 \% \mathrm{~K}$ (Fig. $4 \mathrm{~b}$ and c), one could misinterpret the plateaus in Fig. $4 \mathrm{~b}$ and $\mathrm{c}$ of $\mathrm{D}+10 \% \mathrm{~K}$ as presence of kerosene only. However, this is not the case because the signal magnitude (almost constant) is lower for kerosene ( $\mathrm{K}$ - smooth in Fig. 4a) as compared to D $+10 \% \mathrm{~K}$ (in Fig. $4 \mathrm{~b}$ and c).

Considering both optical sensors, the table model presents a better sensing capability than the portable sensor namely, the signal from $\mathrm{D}+10 \% \mathrm{~K}$ liquid plateaus for both the smooth and rough glass slides of the PS after a few minutes. One would, therefore, assume a constant variation in the liquid behavior. However, for the same liquid, the TM senses the variation in the liquid spreading making it a more sensitive optical sensor. Additionally, in Fig. 4a, the PS signal from kerosene also plateaus after the first few minutes on the smooth glass. On the reverse, the TM signal from the relatively smooth metal surface, $\mathrm{Ra}=0.05 \mu \mathrm{m}$, suggests detectable variations in the liquid drop spreading. Despite the different resolution of the TM and PS sensors, the irregularity of the $\mathrm{D}+10 \% \mathrm{~K}$ is apparent with distinct features in the signal from both devices.

\section{Conclusion}

In this work, we have introduced two novel optical sensors namely, the table model (TM) and portable (PS) sensors, good for monitoring the dynamic change of a liquid drop spreading for shorter and relatively longer duration measurements, respectively. These sensors may have importance such as in the study of hydrophilic and hydrophobic liquids over rough surfaces. The portability and ease of use of the PS make it a useful and robust tool for field applications. The TM, only limited in its portability, rather presents a faster method for the monitoring of the liquid spreading under laboratory conditions. Both approaches also allow the screening of liquid adulterations, such as presented in this study, with high reliability. The liquid drop spreading studied on the rough surfaces by means of the optical sensors reveals that the surface roughness and the complexity of the liquid influence the spreading. Specifically, the contact angle becomes abnormal for the $\mathrm{D}+10 \% \mathrm{~K}$ complex liquid. This abnormal behavior of the contact angle manifests in the spreading of the liquid on rough and smooth surfaces which is detected in the optical signal from both sensors. These monitoring methods can also be applied in the detection of wine adulteration by glycol and the adulteration of strong alcohols such as vodka and water.

\begin{abstract}
Abbreviations
BS: Beam splitter; D: Diesel or Detector; DOE: Diffractive optical element; GF: Ground surface; K: Kerosene; L: Lens; PS: Portable sensor; RI: Refractive index (n); S: Time-dependent or dynamic signal; TM: Table model
\end{abstract}

\section{Acknowledgements}

The authors would like to thank Mr. Tommi Itkonen for his support during the measurements.

Funding

Not Applicable.

Availability of data and materials

Details about data have been provided in the manuscript. 


\section{Authors' contributions}

All sensor measurements, BA; Refractive index and contact angle measurements, BK; Writing-Original draft preparation, BA; Writing-Review\& Editing, BA; BK and K-EP; Supervision of the entire work K-EP. All authors read and approved the final manuscript.

\section{Competing interests}

The authors declare that they have no competing interests.

\section{Publisher's Note}

Springer Nature remains neutral with regard to jurisdictional claims in published maps and institutional affiliations.

Received: 23 July 2018 Accepted: 22 October 2018

Published online: 01 November 2018

\section{References}

1. Rosenholm, J.B., Peiponen, K.E., Gornov, E.: Materials cohesion and interaction forces. Adv. Colloid Interf. Sci. 141, 48-65 (2008)

2. Yuan, Y., Lee, T.R.: Contact angle and wetting properties. In: Bracco, G., Holst, B. (eds.) Surface Science Techniques, pp. 3-34. Springer, Berlin (2013)

3. Johnson, R.E., Dettre, R.H.: Contact angle hysteresis. I. Study of an idealized rough surface. In: Contact Angle, Wettability and Adhesion. Adv. Chem. Ser. 43, pp. 112-135. American Chemical Society, Washington DC (1964)

4. Schulze, R.-D., Possart, W., Kamusewitz, H., Bischof, C.: Young's equilibrium contact angle on rough solid surfaces. Part I. an empirical determination. J. Adhesion Sci. Technol. 3, 39-78 (1989)

5. Wang, X.D., Peng, X.F., Lu, J.F., Liu, T., Wang, B.X.: Contact angle hysteresis on rough solid surfaces. Heat. Tran. Asian. Res. 33, 201-210 (2004)

6. David, R., Neumann, A.W.: Contact angle hysteresis on randomly rough surfaces: a computational study. Langmuir. 29, 4551-4558 (2013)

7. Wolansky, G., Marmur, A.W.: The actual contact angle on a Heterogenous rough surface in three dimensions. Langmuir. 14, 5292-5297 (1998)

8. Prajitno, D.H., Maulana, A., Syarif, D.G.: Effect of surface roughness on contact angle measurement of Nanofluid on surface of stainless steel 304 by sessile drop method. J. Phys. 739(1-5), (2016). https://doi.org/10.1088/ 1742-6596/739/1/012029

9. Quere, D.: Wetting and roughness. Annu. Rev. Mater. Res. 38, 71-99 (2008)

10. Ralston, J., Popescu, M., Sedev, R.: Dynamics of wetting from an experimental point of view. Annu. Rev. Mater. Res. 38, 23-43 (2008)

11. Aytouna, M., Parcedes, J., Shahidzadeh-Bonn, N., Moulinet, S., Wagner, C., Amarouchene, Y.: Drop formation in non-Newtonian fluids. Phys. Rev. Lett. 110, 034501-1-5 (2013)

12. Cottington, R., Murphy, C.M., Singeleterry, C.R.: Effect of polar-nonpolar additives on oil spreading on solids, with applications to non-spreading oils, contact angle, wettability and adhesion. Adv. Chem. 43, 341-354 (1964)

13. Brutin, D.: Droplet Wetting and Evaporation: from Pure to Complex Fluids. Elsevier, Amsterdam (2015)

14. Rafai, S., Sarker, D., Bergeron, V., Meunier, J., Bonn, D.: Superspreading: aqueous surfactant drops spreading on hydrophobic surfaces. Langmuir. 18, 10486-10488 (2002)

15. Tanner, L.: The spreading of silicone oil drops on horizontal surfaces. J. Phys. D: Applied Physics. 12, 1473-1484 (1979)

16. Kanyathare, B., Kuivalainen, K., Räty, J., Silftsten, P., Bawuah, P., Peiponen, K.E.: A prototype of an optical sensor for the identification of diesel oil adulterated with kerosene. J. Eur. Opt. Soc. Rapid. Publ. 14, 1-6 (2018)

17. Kanyathare, B., Peiponen, K.-E.: Wavelength-dependent excess permittivity as indicator of kerosene in diesel oil. Appl. Opt. 57, 2997-3002 (2018)

18. Kanyathare, B., Peiponen, K-E. Hand-held refractometer-based measurement and excess permittivity analysis method for detection of diesel oils adulterated by kerosene in field conditions. Sensors. 18, 1551 (2018)

19. Palik, E.D.: Handbook of Optical Constants of Solids Vol. I-IV. p. 313. Academic Press, San Diego (1985)

20. Tanner, L.H.: The use of laser light in the study of metal surfaces. Opt. Laser. Technol. 8, 113-116 (1976)

21. Hensler, D.H.: Light scattering from fused polycrystalline Aluminium oxide surfaces. Appl. Opt. 11, 2522-2528 (1972)

22. Beckmann, P.: Scattering of Electromagnetic Waves from Rough Surfaces. Macmillan, New York (1963)

23. Fujii, H., Asakura, T.: Roughness measurements of metal surfaces using laser speckle. J. Opt. Soc. Am. 67, 1171-1176 (1977)
24. Silvennoinen, R., Peiponen, K.E., Myller, K.: Specular Gloss. Elsevier, Amsterdam (2008)

25. Niskanen, I., Peiponen, K.E.: Räty: assessment of refractive index of pigments by gaussian fitting of light backscattering data in context of the liquid immersion method. Appl. Spec. 64, 558-561 (2010)

26. Kuivalainen, K., Oksman, A., Juuti, M., Myller, K., Peiponen, K.-E.: Advanced glossmeters for industrial applications. Opt. Rev. 17, 248-251 (2010)

27. Reis, J.C., Iglesias, T.P., Douhéret, G., Davis, M.J.: The permittivity of thermodynamically ideal liquid mixtures and the excess relative permittivity of binary dielectrics. Phys. Chem. 11, 3977-3986 (2009)

28. Rahimi, P., Ward, C.A.: Contact angle hysteresis on smooth and homogenous surfaces in gravitational fields. Microgravity sci. Technol. 16, 231-235 (2005)

29. Raeesi, B., Morrow, N.R., Mason, G.: Contact angle hysteresis at smooth and rough surfaces. In: GeoConvention: Integration, Calgary, Canada, p. 2013

30. Marmur, A.: Contact angle hysteresis on Heterogenous smooth surfaces. J. Colloid. Interface. Sci. 168, 40-46 (1994)

\section{Submit your manuscript to a SpringerOpen ${ }^{\circ}$ journal and benefit from:}

- Convenient online submission

- Rigorous peer review

- Open access: articles freely available online

High visibility within the field

- Retaining the copyright to your article

Submit your next manuscript at $\boldsymbol{\nabla}$ springeropen.com 